\title{
A comparison of efficacy and safety of preoperative versus intraoperative computed tomography-guided thoracoscopic lung resection
}

\author{
Yin-Kai Chao, MD, ${ }^{\mathrm{a}}$ Kuang-Tse Pan, MD, ${ }^{\mathrm{b}}$ Chih-Tsung Wen, MD, ${ }^{\mathrm{a}}$ Hsin-Yueh Fang, MD, ${ }^{\mathrm{a}}$ and \\ Ming-Ju Hsieh, MD ${ }^{\mathrm{a}}$
}

\begin{abstract}
Background: The efficacy and safety of intraoperative computed tomography (IOCT)-guided lung tumor localization and resection performed in a hybrid operating room (OR) compared with the conventional 2-stage preoperative CT (POCT)-guided approach for the treatment of small and deep solitary pulmonary nodules (SPNs) remains unknown.
\end{abstract}

Methods: We compared IOCT-guided (IOCT group) and POCT-guided (POCT group) thoracoscopic resections in 64 consecutive patients with SPNs. The main outcome measures included efficacy, safety, and radiation exposure.

Results: The IOCT $(\mathrm{n}=34)$ and POCT $(\mathrm{n}=30)$ groups had a similar SPN depth-to-size ratio. All SPNs were successfully localized and removed using a minimally invasive approach. There were no significant intergroup differences in localization procedural time (mean, 17.68 [IOCT] vs 19.63 minutes [POCT]; $P=.257$ ) and radiation exposure (median, 3.65 [IOCT] vs $6.88 \mathrm{mSv}$ [POCT]; $P=.506)$. The use of a hybrid operating room (OR) for tumor localization significantly reduced the patient time at risk (ie, the interval from completion of localization to skin incision; mean, 215.83 [POCT] vs 13.06 minutes $[\mathrm{IOCT}] ; P<.001)$. However, the IOCT-guided approach significantly increased the time under general anesthesia (mean, 120.61 [POCT] vs 163.1 minutes [IOCT]; $P<.001$ ) and the total OR utilization time (mean, 168.68 [POCT] vs 227.41 minutes [IOCT]; $P<.001$ ).

Conclusions: Compared with the POCT-guided approach, the IOCT-guided approach decreased the time at risk, despite a significant increase in the global OR utilization time. Because no significant outcome differences were evident, the choice between the 2 approaches should be based on the most readily available approach at a surgeon's specific facility. ( $\mathrm{J}$ Thorac Cardiovasc Surg $2018 ; 156: 1974-83)$

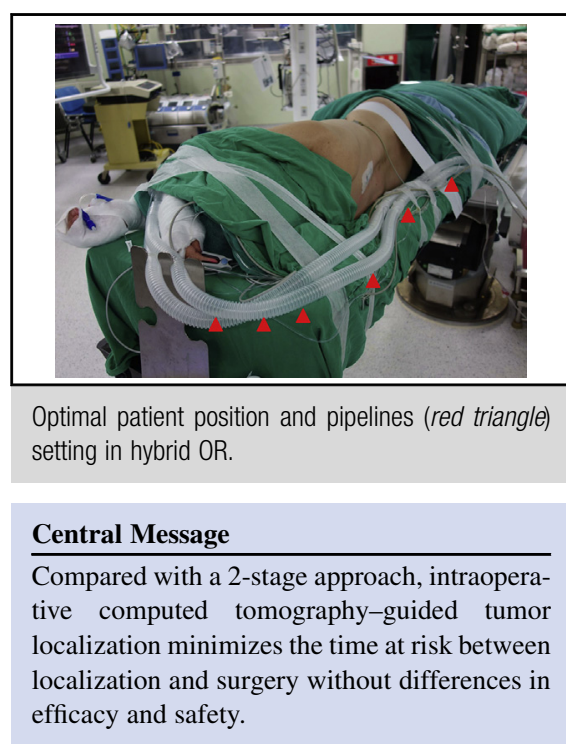

\section{Perspective}

Compared with the preoperative computed tomography-guided approach, the 2-stage approach was associated with a decreased time at risk. However, the global operating room utilization time increased significantly. Because no significant outcome differences were evident, the choice between the 2 approaches should be based on the most readily available approach at a surgeon's specific facility.

See Editorial Commentary page 1984.

See Editorial page 1970.
From the ${ }^{\mathrm{a}}$ Division of Thoracic Surgery, Chang Gung Memorial Hospital; and ${ }^{\mathrm{b}} \mathrm{De}-$ partment of Medical Imaging and Intervention, College of Medicine, Chang Gung University, Taoyuan, Taiwan.

Supported by a grant (CMRPG3F1813) from the Chang Gung Memorial Hospital, Taiwan.

Received for publication Jan 4, 2018; revisions received June 10, 2018; accepted for publication June 17, 2018; available ahead of print Aug 14, 2018.

Address for reprints: Yin-Kai Chao, MD, Division of Thoracic Surgery, Chang Gung Memorial Hospital-Linko, Chang Gung University, Taoyuan, Taiwan (E-mail: chaoyk@cgmh.org.tw).

$0022-5223 / \$ 36.00$

Copyright (c) 2018 by The American Association for Thoracic Surgery

https://doi.org/10.1016/j.jtcvs.2018.06.088
The increasing use of low-dose computed tomography (CT) for lung cancer screening has led to the identification of a high number of solitary pulmonary nodules (SPNs). As a consequence, thoracic surgeons are often faced with the removal of SPNs, which are small and can be deeply located

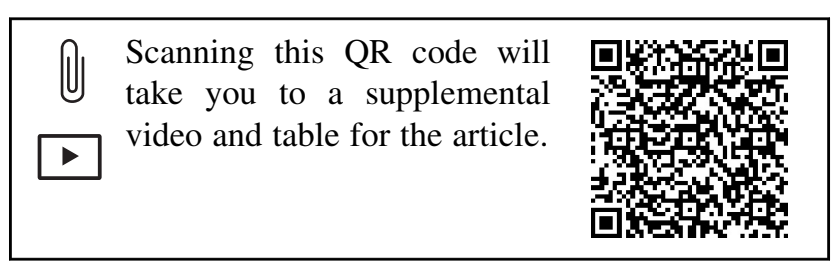



Abbreviations and Acronyms
$\mathrm{CBCT}=$ cone-beam computed tomography
$\mathrm{CI}=$ confidence interval
$\mathrm{CT}=$ computed tomography
$\mathrm{GGN}=$ ground glass nodule
IOCT = intraoperative computed tomography
$\mathrm{IQR}=$ interquartile range
MDCT $=$ multidetector computed tomography
$\mathrm{OR}=$ operating room
POCT $=$ preoperative computed tomography
SPN = solitary pulmonary nodule
TLD $=$ thermoluminescent dosimeter
VATS $=$ video-assisted thoracoscopic surgery

in the lung parenchyma. ${ }^{1}$ Unfortunately, such SPNs are generally not visible to the naked eye and are unlikely to be palpable through thoracoscopic instruments. Suzuki et $\mathrm{al}^{2}$ previously reported a $63 \%$ conversion rate from video-assisted thoracoscopic surgery (VATS) to thoracotomy when lung nodules are less than $10 \mathrm{~mm}$ in diameter or located more than $5 \mathrm{~mm}$ below the pleural surface.

In an effort to minimize the likelihood of unplanned conversion to thoracotomy, different marking methods-including percutaneous CT-guided, ${ }^{3-5}$ bronchoscopically guided (based on segmental anatomy and virtual imaging), ${ }^{6,7}$ and electromagnetic navigation bronchoscopy-guided ${ }^{8-10}$ approaches-have been proposed before embarking on VATS exploration. A randomized study supported the clinical utility of preoperative tumor localization; accordingly, it was significantly associated with a higher rate of successful VATS wedge resection, a decreased operation time, and a lower use of staples, without increasing total costs (compared with no localization). ${ }^{11}$

As far as percutaneous CT-guided SPNs localization is concerned, the most common workflow comprises an initial preoperative CT (POCT)-guided lesion localization (performed in an interventional CT suite) followed by patient transfer to an operating room (OR). ${ }^{3}$ Notably, this 2-stage workflow requires optimized timing and a strict coordination between the CT suite and the OR. Accordingly, longer waiting times portend an increased risk of complications (including pneumothorax, hemothorax, wire dislodgement, and dye fading).

Recently, intraoperative CT (IOCT)-guided VATS performed in a hybrid OR has been proposed to overcome the known shortcomings of the 2-stage approach. Although there are several studies showing the feasibility of the IOCT approach, ${ }^{12-15}$ a direct comparison with the 2-stage approach in terms of accuracy of tumor localization, occurrence of complications, and radiation exposure has never been attempted. We therefore designed the current single-center study to address these knowledge gaps.

\section{MATERIALS AND METHODS \\ Study Patients}

This was an institutional review board-approved (CGMH-IRB 201600671A3) study aimed at comparing IOCT-guided VATS (IOCT group) with the traditional 2-stage approach (POCT group) for SPN localization and removal. Consecutive patients with undiagnosed lung nodules who required tumor localization before surgical resection between April 1, 2017, and October 1, 2017, were deemed eligible. Patients who had more than one nodule requiring localization $(n=7)$ or refused to give informed consent $(\mathrm{n}=2)$ were excluded. Figure 1 summarizes the flow of patients based on their surgical allocation.

\section{Indications for Tumor Localization and Selection of the Localization Method}

When solid nodules were present, localization was recommended for small (diameter $<10 \mathrm{~mm}$ ) or deeply located (distance from the visceral pleura $>10 \mathrm{~mm}$ ) SPNs. Subpleural cavitary lesions and ground glass nodules (GGNs) were localized regardless of their size and depth. POCTguided localization was introduced in our hospital in 2007, and it has been adopted as the standard approach thereafter. ${ }^{16}$ Our IOCT-guided approach was commenced in 2016. When the hybrid OR (which is shared with the cardiovascular department) was available, patients in need of tumor localization underwent the IOCT approach. If this was not the case, the POCT approach was used.

\section{POCT-Guided Localization}

A single board-certified radiologist (K-T.P.) experienced in interventional techniques performed CT-guided localization. Positioning of patients in the CT scanner (GE HiSpeed, Milwaukee, Wisc) was performed to achieve the shortest direct path from the skin to the SPN. Images acquired to localize the lesion were $2.5 \mathrm{~mm}$ thick. When possible, we used a direct and vertical needle trajectory to reach the target lesion. At the site of puncture, a careful skin cleansing process was performed. Using local anesthesia, we created a small skin incision with a scalpel and gradually inserted a $10.7-\mathrm{cm}$-long, 20-gauge cannula needle housing a $20-\mathrm{cm}$ long double-thorn hook wire (DuaLok; Bard Peripheral Vascular, Tempe, Ariz) through the chest wall. The procedure was performed under sequential CT guidance. When possible, lung lesions were pierced through the cannula needle. When the needle tip was properly positioned within the lesion or in its close proximity, we advanced the hook wire along the cannula. Superficial lesions were localized through the injection of patent blue vital dye $(0.5 \mathrm{~mL}$, patent blue V $2.5 \%$; Guerbet, Aulnay-sous-Bois, France) with a 22 -gauge spinal needle (length: $8.9 \mathrm{~cm}$ ). Correct positioning of the hook wire with respect to the lung nodule was confirmed through immediate follow-up CT scans. Patients were then transferred to the general ward to wait for surgery.

\section{IOCT-Guided Localization}

IOCT-guided localization was performed in a hybrid OR (Figure 2, $A$ ) in which a C-arm cone-beam CT (CBCT; ARTIS zeego; Siemens Healthcare, Erlangen, Germany) and a Magnus surgical table (Maquet Medical Systems, Wayne, NJ) were available. A single team of thoracic surgeons performed both localization and surgery. The procedural workflow has been previously described in detail. ${ }^{17}$ Patients were placed in the lateral decubitus position after induction of general anesthesia (Figure 2, B). During end-inspiratory breath-holding, an initial scan was acquired for surgical planning using a 6-second acquisition protocol (6s DynaCT Body). The entering trajectory was modeled in the isotropic data set under the syngo 


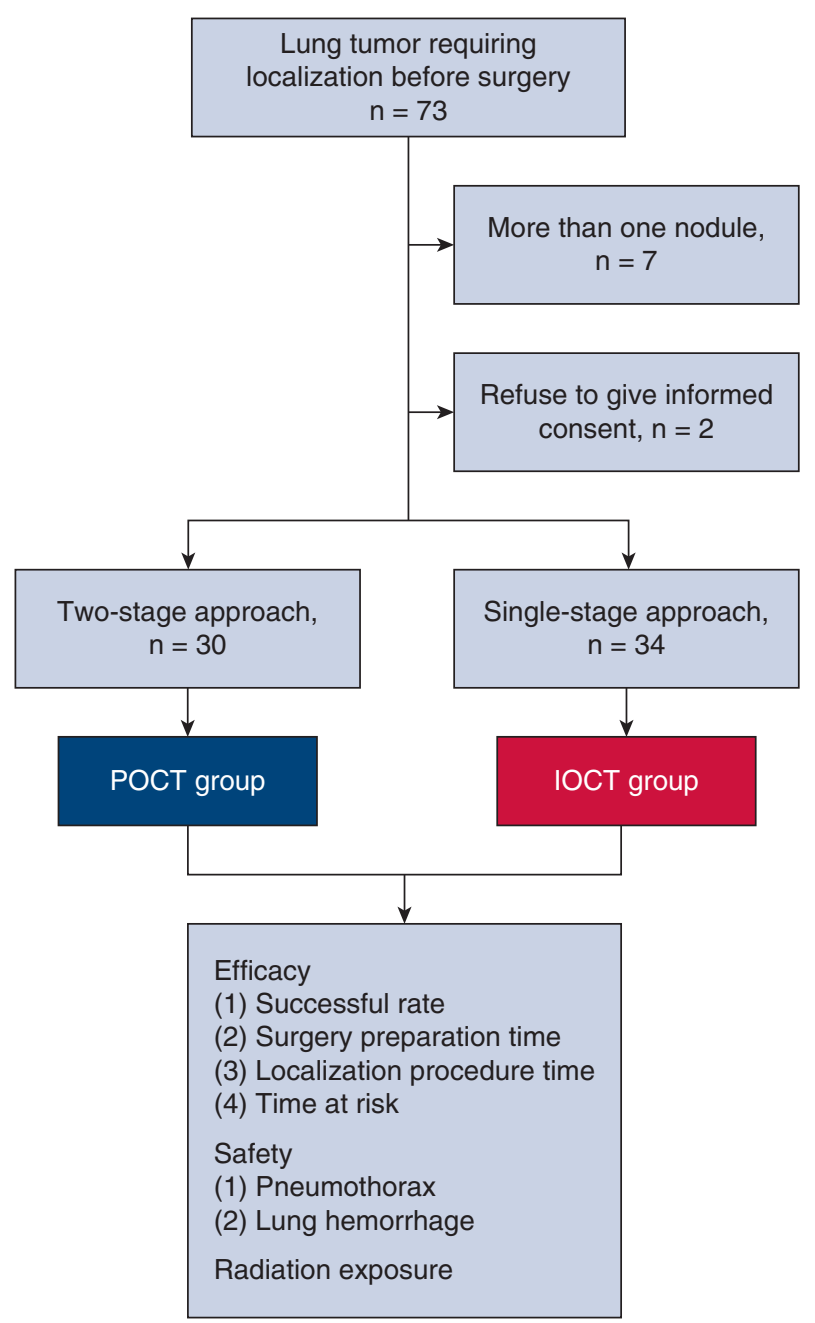

FIGURE 1. Flow of the patients through the study. POCT, Preoperative computed tomography; IOCT, intraoperative computed tomography.

Needle Guidance of a syngo X-Workplace (Siemens Healthcare). We initially laid out the needle trajectory by marking the entry and the target points (Figure $3, A$ ); the needle entry point and angulation were visualized by projecting a laser-target cross onto the patient's surface (Figure 3,B). Under three-dimensional laser-guidance and guided fluoroscopy, we introduced an 18-gauge marker needle into the patient's thorax during end-inspiratory breath-holding. We then corrected both needle orientation and positioning by projecting the planned, virtual needle trajectory onto the live fluoroscopic image. A fluoroscopic bulls-eye approach was used to introduce the needle and guide it to the projected target. When the lesion was reached, the tumor was localized by placing a localization wire (DuaLok; Bard Peripheral Vascular). Superficial lesions were delineated by injecting patent blue vital dye $(0.3-0.5 \mathrm{~mL}$, patent blue V $2.5 \%$; Guerbet). Postprocedural CBCT scans were obtained to confirm the accuracy of tumor localization (Figure 3,C).

\section{Surgical Treatment}

After VATS wedge resection (conducted either under hook wire or dye guidance), frozen section examination of the resected lesion was performed (Figure 3,D). Patients with a confirmed diagnosis of primary lung cancer underwent lobectomy. Patients with peripheral lung cancer of limited size $(<2 \mathrm{~cm})$ and adequate resection margins (either $>2 \mathrm{~cm}$ or greater than the tumor size) were treated with a sublobar resection.

\section{Outcome Assessment}

The primary outcome measures included (1) the rate of successful targeting during localization (defined as the number of successful targeting procedures divided by the number of all localization procedures) and (2) the rate of successful localization in the operating field (defined as the number of successful targeting procedures minus the number of wire dislodgements or dye fading or spillage occurring in the operation field divided by the number of all localization procedures). The following parameters served as secondary outcome measures: (1) time elapsed for tumor localization, (2) time for surgical preparation, and (3) time at risk. The procedural time for localization was defined as the time between the start of the preprocedural $\mathrm{CT}$ scan to the termination of the postprocedural $\mathrm{CT}$ scan in the POCT group and as the time between the docking of the Carm to the end of the localization procedure (ie, retraction of the $\mathrm{C}$-arm from the table to the park position) in the IOCT group. The time for surgical preparation was defined as the time between completion of the general anesthesia and skin incision in the POCT group and as the time between completion of general anesthesia and C-arm docking plus $\mathrm{C}$-arm parking to skin incision in the ICOT group. The time at risk was defined as the time between the completion of localization and skin incision. Besides localization-related time parameters, we also compared the following variables: (1) time to treat, defined as the time interval from the date of diagnosis to the date of surgery; (2) operation time; (3) length of time under anesthesia; (4) global operating room utilization time; and (5) length of hospital stay.

Complications were subdivided into 2 categories (ie, pneumothorax and lung hemorrhage), and their occurrence was recorded after the immediate follow-up CT scans following localization. In line with the 2010 British Thoracic Society guidelines, large or small pneumothorax was defined by distance between lung margin and chest wall greater or less than $2 \mathrm{~cm}$, respectively. ${ }^{18}$

\section{Radiation Monitoring}

To quantify the radiation surface dose to patients, 4 sets of thermoluminescent dosimeters (TLDs; UD-802A; Panasonic, Osaka, Japan) were placed around the patient's chest wall at the lesion level. The mean values measured by the 4 TLDs were used for analysis. The radiation dose absorbed by each TLD was measured using a TLD reader (UD-716AGL TLD reader; Panasonic).

\section{Statistical Analysis}

Normally distributed continuous variables were presented as means ( $95 \%$ confidence interval $[\mathrm{CI}]$ ) and compared with a 2-sample (unpaired) Student $t$ test. Skewed variables are summarized as medians (interquartile range [IRQ]) and compared using the Mann-Whitney $U$ test. Categorical data were reported as counts and percentages and analyzed with the $\chi^{2}$ test or the Fisher exact test (if a cell value was lower than 5). All calculations were performed using the IBM SPSS 22.0 statistical package (IBM, Armonk, NY). All $P$ values were 2 -tailed, with $P<.05$ being considered statistically significant.

\section{RESULTS}

\section{Characteristics of Patients and Pulmonary Nodules}

Table 1 depicts the general characteristics of the study participants. The IOCT and POCT groups consisted of 34 and 30 patients, respectively. Although the age distribution was similar, a higher number of patients in the IOCT group had a more advanced American Society of Anesthesiologists classification. Based on the preoperative CT findings, 32 $(50 \%)$ lung nodules were categorized as solid lesions, whereas $32(50 \%)$ were classified as GGNs. The mean 

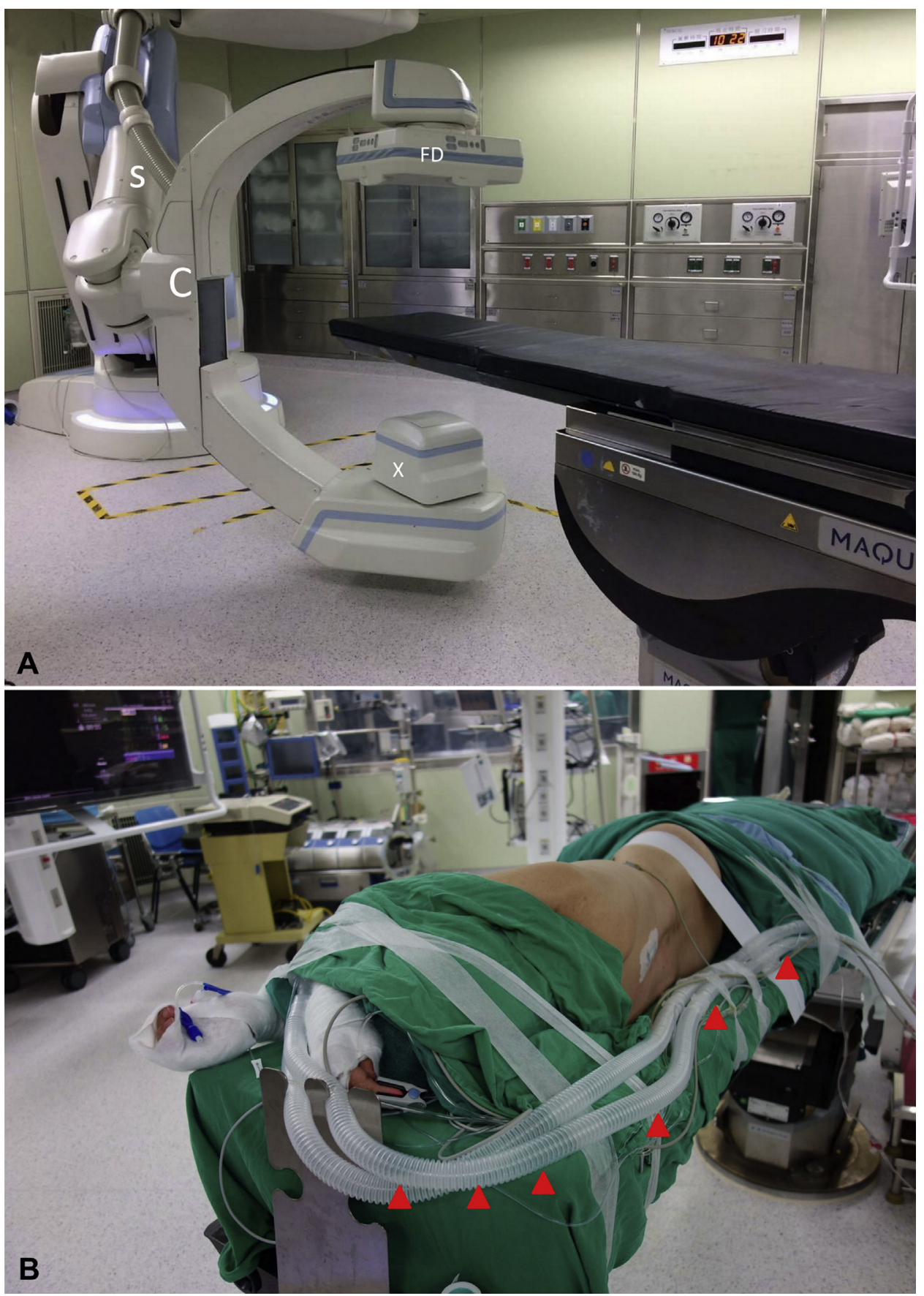

FIGURE 2. A, Hybrid OR equipped with a floor-mounted robotic C-arm cone-beam computed tomography (ARTIS zeego; Siemens Healthcare, Erlangen, Germany) and a Magnus surgical table (Maquet Medical Systems, Wayne, NJ). B, Patient placed in the lateral decubitus position. All pipelines (red triangle) in the anesthesia side were gathered and aligned within the edge of the table to avoid entanglements with the rotating $\mathrm{C}$-arm. $S, \mathrm{Stand}$ arm; $C, \mathrm{C}$-arm; $F D$, flat detector; $X$, X-ray tube assembly with primary collimator.

size of SPNs on preoperative CT images was $6.85 \mathrm{~mm}$ (range, 6.14-7.56 mm) with a similar side distribution. Their median distance from the pleural surface was $7.75 \mathrm{~mm}$ (range, 2.25-15 mm), whereas the median tumor depth-tosize ratio was $1.08(0.5-2.33)$. Table E1 summarizes the size and depth distribution of the 64 lung tumors classified according to their appearance (GGN and solid subgroups).
The interval between diagnosis and surgery (time to treat) did not show significant intergroup differences.

\section{Localization Procedure}

Table 2 summarizes the technical details of the localization procedure. All 34 patients in the IOCT group underwent localization in the lateral decubitus position. In the 

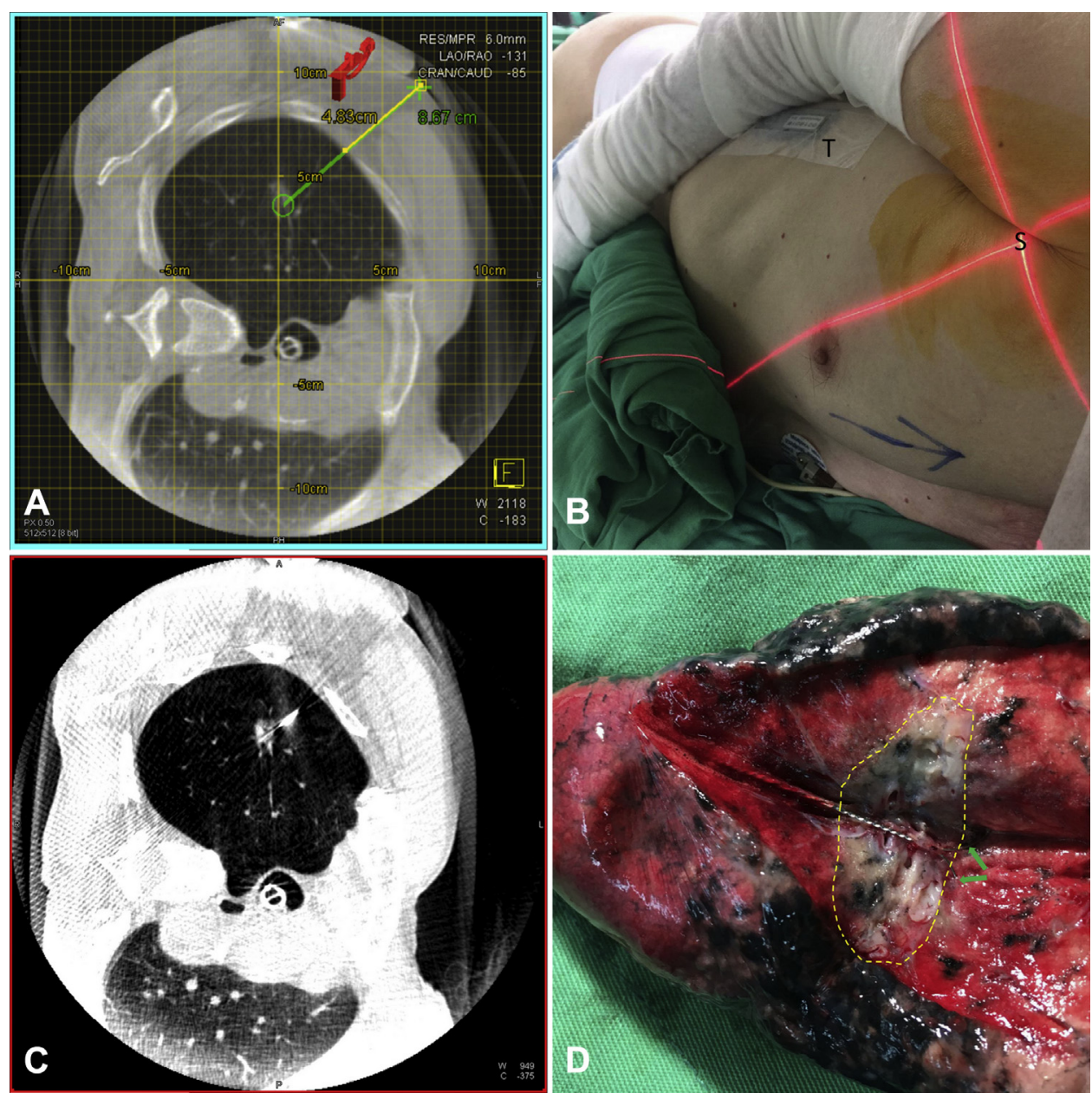

FIGURE 3. A, Image-guided needle pathway planning (green line) based on cone-beam computed tomography (CBCT) imaging. B, Needle localization through the planned pathway as projected by the laser beam. The laser-targeting cross is projected onto the patient's surface, providing an indication for both the needle skin entry point $(\mathrm{S})$ and angulation. A thermoluminescent dosimeter $(\mathrm{T})$ was placed on the patient's chest for measuring the radiation exposure. $\mathrm{C}$, CBCT image obtained after needle puncture (before wire deployment). D, Surgical specimen showing the penetration of the hook wire through the lesion (yellow dotted line), with the hook tip (green arrow) at the bottom of the lesion.

POCT group, localization was performed using the supine and prone positions in $17(57 \%)$ and $13(43 \%)$ patients, respectively. All lung nodules were identifiable on either CBCT or multidetector computed tomography (MDCT). Localization was performed either by hook wire $(\mathrm{n}=32 ; 50 \%)$ or dye $(\mathrm{n}=32 ; 50 \%)$ and was successful in all patients (rate of successful targeting during localization: 100\%). Figure 4 illustrates the definitions and the results of the index time parameters in the two groups. The IOCT group required a longer preparation time in the OR (defined as the sum of intervals between the completion of anesthesia to the beginning of localization plus the completion of localization to the skin incision) than the POCT group did (from the completion of anesthesia to the skin incision). The mean localization procedural time (IOCT, 17.68 minutes; POCT group, 19.63 minutes; $P=.257$ ) and the median radiation exposure (IOCT, $3.65 \mathrm{mSv}$; POCT group,
$6.88 \mathrm{mSv} ; P=.506$ ) did not differ significantly between the 2 groups. The use of IOCT significantly reduced the time at risk (defined as the interval from the completion of localization to skin incision; mean, 215.83 [POCT] vs 13.06 minutes [IOCT]; $P<.001$ ). However, the time under general anesthesia and the global OR utilization time were significantly longer in the IOCT group $(P<.001)$.

Regarding complications after localization, 15 patients $(23.4 \%)$ patients had pneumothorax and 5 patients $(7.8 \%)$ had lung parenchyma hemorrhage demonstrated on postprocedural CBCT or CT findings. The occurrence rate of pneumothorax was significantly lower in the IOCT group $(2.9 \%)$ than in the POCT group $(46.7 \% ; P<.001)$. All cases of pneumothorax were small (distance between the chest wall and the lung margin $<2 \mathrm{~cm}$ ), and neither drainage before surgery nor blood transfusions were required. 
TABLE 1. General characteristics of the study patients

\begin{tabular}{|c|c|c|c|c|}
\hline & Entire cohort & POCT group & IOCT group & $P$ value \\
\hline Number of patients & 64 & 30 & 34 & NA \\
\hline Age, years; mean $(95 \% \mathrm{CI})$ & $55.80(52.87-58.73)$ & $54.63(50.31-58.95)$ & $56.82(52.65-61.0)$ & .461 \\
\hline $\begin{array}{l}\text { Sex } \\
\text { Male } \\
\text { Female }\end{array}$ & $\begin{array}{l}31(48.4 \%) \\
33(51.6 \%)\end{array}$ & $\begin{array}{l}10(33.3 \%) \\
20(66.7 \%)\end{array}$ & $\begin{array}{l}21(61.8 \%) \\
13(38.2 \%)\end{array}$ & .027 \\
\hline $\begin{array}{l}\text { ASA physical status classification } \\
\text { I \& II } \\
\text { III }\end{array}$ & $\begin{array}{l}11(17.2 \%) \\
53(82.8 \%)\end{array}$ & $\begin{array}{r}9(30 \%) \\
21(70 \%)\end{array}$ & $\begin{array}{c}2(5.9 \%) \\
32(94.1 \%)\end{array}$ & .018 \\
\hline $\begin{array}{l}\text { Time to treat, days } \\
\text { Median (IQR) }\end{array}$ & $12(6-17)$ & $12.5(6-16.25)$ & $12(6.5-19)$ & .350 \\
\hline $\begin{array}{l}\text { CT findings } \\
\text { Solid nodule } \\
\text { GGN }\end{array}$ & $\begin{array}{l}32(50 \%) \\
32(50 \%)\end{array}$ & $\begin{array}{l}17(56.7 \%) \\
13(43.3 \%)\end{array}$ & $\begin{array}{l}15(44.1 \%) \\
19(55.9 \%)\end{array}$ & .453 \\
\hline Lesion size on CT, mm; mean $(95 \% \mathrm{CI})$ & $6.85(6.14-7.56)$ & $6.45(5.25-7.65)$ & $7.19(6.33-8.06)$ & .3 \\
\hline $\begin{array}{l}\text { Lesion location } \\
\text { Right-sided } \\
\text { Left-sided }\end{array}$ & $\begin{array}{l}42(65.6 \%) \\
22(34.4 \%)\end{array}$ & $\begin{array}{r}21(70 \%) \\
9(30 \%)\end{array}$ & $\begin{array}{l}21(61.8 \%) \\
13(38.2 \%)\end{array}$ & .6 \\
\hline $\begin{array}{l}\text { Distance to the pleural space, } \mathrm{mm} \\
\text { Median (IQR) }\end{array}$ & $7.75(2.25-15)$ & $7.75(1.5-15)$ & $8(2.75-16.25)$ & .802 \\
\hline $\begin{array}{l}\text { Depth-to-size ratio } \\
\text { Median (IQR) }\end{array}$ & $1.08(0.5-2.33)$ & $0.98(0.38-2.80)$ & $1.13(0.46-2.33)$ & .802 \\
\hline
\end{tabular}

Data are given as counts (percentages in parentheses) or mean (standard deviations in parentheses). POCT, Preoperative computed tomography; IOCT, intraoperative computed tomography; $C I$, confidence interval; $A S A$, American Society of Anesthesiologists; $I Q R$, interquartile ratio; $C T$, computed tomography; GGN, ground glass nodule.

\section{Operative and Perioperative Results}

Upon thoracoscopic exploration, failure of localization occurred in 5 patients (dye spillage [n $=1$ ], dye fading $[\mathrm{n}=2]$, wire dislodgement $[\mathrm{n}=2]$ ), without significant intergroup differences (successful targeting rates during operation: IOCT [91.2\%], POCT [93.3\%]; $P=.302$ ). Failure of localization did not affect the success of VATS resection (nodule localization guided by the lung puncture site). All nodules were resected successfully using VATS, and no conversion to thoracotomy was required. Wedge resection was initially conducted in all patients. In the 27 patients with primary lung malignancies confirmed by intraoperative frozen section and having a resection margin less than $20 \mathrm{~mm}$ (or less than the tumor diameter), an additional pulmonary resection was performed. Six patients $(9.3 \%)$ underwent VATS segmentectomy, whereas 1 patient $(2.2 \%)$ underwent VATS lobectomy. In the presence of an intraoperative frozen-section diagnosis of malignancies or precancerous lesions, an additional systematic lymph node dissection was performed. Twenty-one patients had benign lesions; specifically, the diagnostic distribution was as follows: granulomatous inflammation $(\mathrm{n}=9)$, hamartoma $(n=1)$, organizing pneumonia $(n=7)$, and presence of an intrapulmonary lymph node $(n=4)$. The median hospital stay after operation was 4.5 days, without significant differences between the IOCT and POCT groups $(P=.452)$.

\section{DISCUSSION}

To our knowledge, this is the first study to compare IOCT-guided VATS with the conventional 2-stage POCTguided approach for localization and resection of SPNs. Our data indicate that IOCT-guided VATS performed as well as the conventional POCT approach, with identical successful targeting rates $(100 \%)$ for tumor localization and similar localization time. Owing to the advantage offered by the hybrid OR, the use of IOCT for tumor localization allows surgery to be completed in a timely fashion (mean time: 13.1 vs 215.8 minutes, $P<.001$ ) after lesion localization (thereby minimizing the time at risk). Our findings suggest that IOCT-guided VATS may offer a more patient-centered surgical approach and can serve as a standard approach for treating SPNs which requiring localization.

In the current study, lung lesions were localized using a robotic C-arm CBCT in a hybrid OR. Besides C-arm $\mathrm{CBCT}$, other imaging modalities previously used to localize lung lesions in a hybrid OR include $\mathrm{MDCT}^{19}$ and mobile $\mathrm{O}$ arm CBCT. ${ }^{20}$ We believe that robotic C-arm CBCT-guided tumor localization offers several potential advantages over both MDCT and mobile O-arm CBCT. First, the multiaxial, robotic technology-based system provides an unprecedented flexibility, allowing localization and surgery to be performed on the same table (ie, without the need of patient transfer within the intra-hybrid OR), an opportunity which 
TABLE 2. Surgical variables of the study patients

\begin{tabular}{|c|c|c|c|c|}
\hline & Entire cohort & POCT group & IOCT group & $P$ value \\
\hline Number of patients & 64 & 30 & 34 & NA \\
\hline Induction time, min; mean $(95 \% \mathrm{CI})$ & $33.64(29.58-37.70)$ & $24.90(20.09-29.71)$ & $41.35(36.09-46.61)$ & $<.001$ \\
\hline Preparation time, min; mean $(95 \% \mathrm{CI})$ & $28.67(25.45-31.89)$ & $25.27(21.02-29.51)$ & $31.68(26.95-36.40)$ & .046 \\
\hline Localization time, min; mean $(95 \% \mathrm{CI})$ & $18.59(16.89-20.30)$ & $19.63(17.31-21.96)$ & $17.68(15.13-20.23)$ & .257 \\
\hline Time at risk, min, mean $(95 \% \mathrm{CI})$ & $108.11(77.43-138.79)$ & $215.83(178.20-253.46)$ & $13.06(11.53-14.59)$ & $<.001$ \\
\hline $\begin{array}{l}\text { Patient position for localization } \\
\text { Supine or prone } \\
\text { Lateral decubitus }\end{array}$ & $\begin{array}{l}30(46.9 \%) \\
34(53.1 \%)\end{array}$ & $\begin{array}{c}30(100 \%) \\
0\end{array}$ & $\begin{array}{c}0 \\
34(100 \%)\end{array}$ & $<.001$ \\
\hline $\begin{array}{l}\text { Localization technique } \\
\text { Hook wire } \\
\text { Dye }\end{array}$ & $\begin{array}{l}32(50 \%) \\
32(50 \%)\end{array}$ & $\begin{array}{l}13(43.3 \%) \\
17(56.7 \%)\end{array}$ & $\begin{array}{l}19(55.9 \%) \\
15(44.1 \%)\end{array}$ & .001 \\
\hline $\begin{array}{l}\text { Procedural complications } \\
\text { Pneumothorax } \\
\text { Lung hemorrhage }\end{array}$ & $\begin{array}{c}15(23.4 \%) \\
5(7.8 \%)\end{array}$ & $\begin{array}{r}14(46.7 \%) \\
4(13.3 \%)\end{array}$ & $\begin{array}{l}1(2.9 \%) \\
1(2.9 \%)\end{array}$ & $\begin{array}{c}<.001 \\
.141\end{array}$ \\
\hline $\begin{array}{l}\text { TLD dose, } \mathrm{mSv} \\
\text { Median (IQR) }\end{array}$ & $5.12(1.85-12.18)$ & $6.88(2.80-32.24)$ & $3.65(1.13-10.68)$ & .506 \\
\hline $\begin{array}{l}\text { Operative procedure } \\
\text { Segmentectomy } \\
\text { Wedge resection } \\
\text { Lobectomy }\end{array}$ & $\begin{array}{c}6(9.3 \%) \\
57(89.1 \%) \\
1(1.6 \%)\end{array}$ & $\begin{array}{c}4(13.3 \%) \\
26(86.7 \%) \\
0\end{array}$ & $\begin{array}{c}2(5.9 \%) \\
31(91.2 \%) \\
1(2.9 \%)\end{array}$ & .394 \\
\hline $\begin{array}{l}\text { Final pathological diagnosis } \\
\text { Primary lung cancer } \\
\text { Lung metastases } \\
\text { Benign nodule }\end{array}$ & $\begin{array}{l}27(42.2 \%) \\
16(25.0 \%) \\
21(32.8 \%)\end{array}$ & $\begin{array}{r}11(36.7 \%) \\
8(26.7 \%) \\
11(36.7 \%)\end{array}$ & $\begin{array}{r}16(47.1 \%) \\
10(29.4 \%) \\
8(23.5 \%)\end{array}$ & 695 \\
\hline Rate of successful targeting during localization & $64(100 \%)$ & $30(100 \%)$ & $34(100 \%)$ & NA \\
\hline Rate of successful targeting during operation & $59(92.2 \%)$ & $28(93.3 \%)$ & $31(91.2 \%)$ & .302 \\
\hline Operation time, min; mean $(95 \% \mathrm{CI})$ & $86.93(77-96.86)$ & $80.50(66.54-94.46)$ & $93.14(78.55-107.72)$ & .205 \\
\hline Length of time under anesthesia, min; mean $(95 \% \mathrm{CI})$ & $142.23(130.11-154.35)$ & $120.61(103.96-137.25)$ & $163.10(148.47-177.73)$ & $<.001$ \\
\hline Global operation room time, min; mean $(95 \% \mathrm{CI})$ & $198.56(185.43-211.70)$ & $168.68(153.52-183.83)$ & $227.41(211.85-242.98)$ & $<.001$ \\
\hline $\begin{array}{l}\text { Length of hospital stay, days } \\
\text { Median (IQR) }\end{array}$ & $4.5(4-5.75)$ & $5(4-6)$ & $4(4-5)$ & .452 \\
\hline
\end{tabular}

Data are given as counts (percentages in parentheses) or means (standard deviations in parentheses). POCT, Preoperative computed tomography; IOCT, intraoperative computed tomography; $C I$, confidence interval; $T L D$, thermoluminescent dosimeter; $I Q R$, interquartile ratio.

is less likely when MDCT is used in hybrid OR. Although mobile O-arm CBCT may theoretically offer a similar advantage, the open gantry design of $\mathrm{C}$-arm CBCT is comparatively characterized by a higher positioning flexibility for lesion targeting (which allowed us to successfully complete the localization procedure in the decubitus position, with no need to change the patient position). Importantly, the iGuide navigation software incorporated in this system provides two additional advantages, including (1) the possibility to point out the needle entry point through a laser cross upon determination of the needle path based on preprocedural CBCT data, and (2) an accurate visualization of the virtual needle pathway when approaching the target lesion through fluoroscopy. These 2 features actually eliminate the need for repeated scans during needle targeting. All these features contribute to the higher efficacy of C-arm CBCT for IOCT-guided VATS over other imaging modalities.

However, one of the major shortcomings we faced during the development of our IOCT-guided VATS protocol was the possible collision between the rotating $\mathrm{C}$-arm and the surgical table. It should be noted that the field of view $(24 \times 18.5 \mathrm{~cm}$, DynaCT mode $)$ provided by CBCT is smaller than that of MDCT. To include both the target lung lesion and the needle entry site into the same CBCT field of view, the surgical table might be placed too low and collide with the $\mathrm{C}$-arm. This concrete risk is especially evident for patients with peripheral lung masses or large chest cavities. Our previous study showed that a collision between the rotating $\mathrm{C}$-arm and the surgical table can 


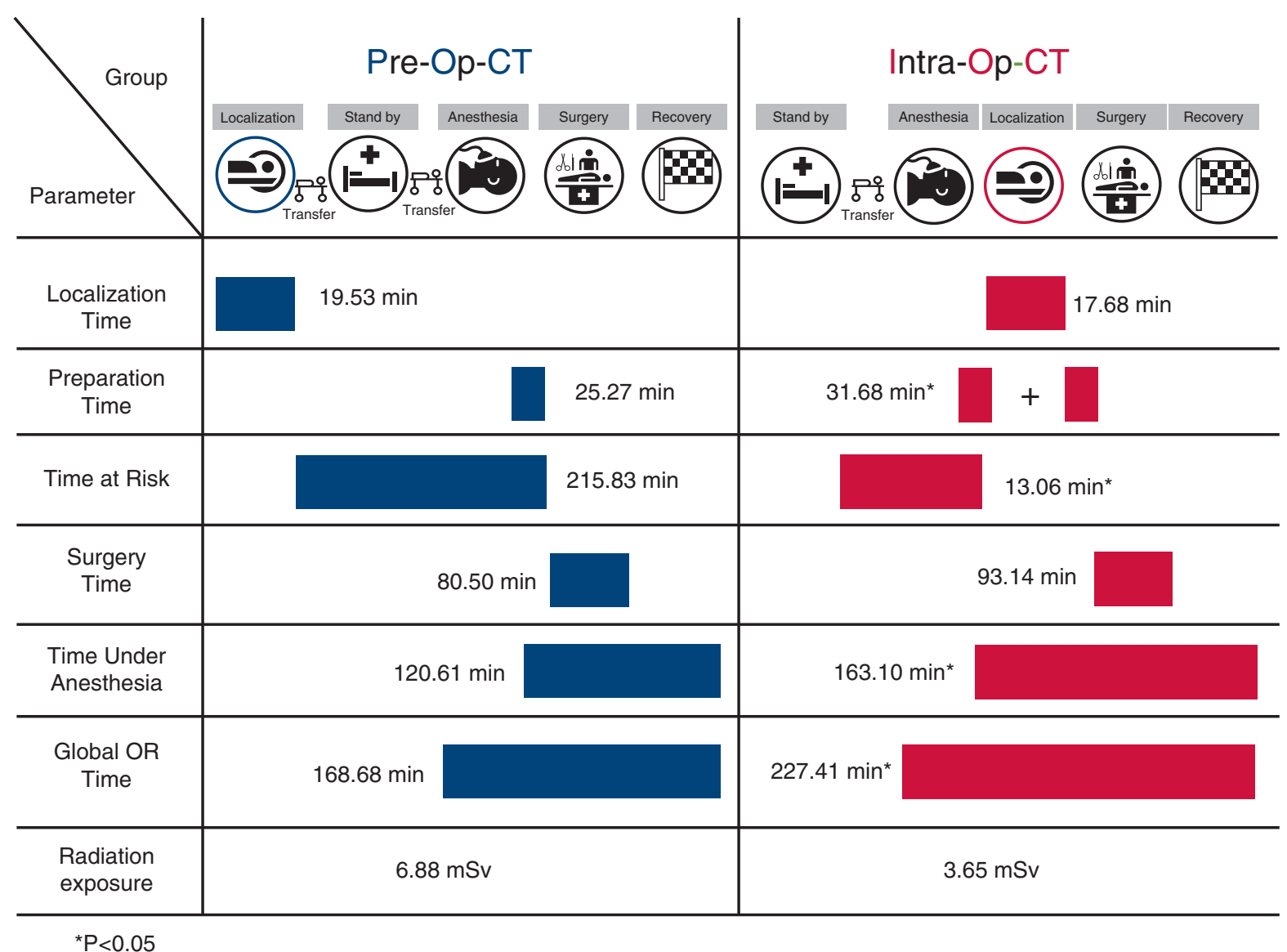

FIGURE 4. Graphical representation of the index time parameters in the 2 study groups. Pre-Op-CT, Preoperative computed tomography; Intra-Op-CT, intraoperative computed tomography.

occur in up to $40 \%$ of patients treated with IOCT-guided VATS. ${ }^{14}$ When this is the case, the patient should be repositioned in the supine or prone position to complete the localization procedure, ultimately resulting in a longer procedural time ( $>40$ minutes). A reduction of the collision rate was successfully achieved after treating our initial 30 patients with IOCT-guided VATS (owing to a learning curve effect). ${ }^{15}$ This positive achievement was attributable to our increased confidence in the reciprocal positioning of the patient, surgical table, and C-arm. ${ }^{21}$ We have previously reported in detail the optimal setup to be used in a hybrid OR; specifically, we identified 8 different settings based on 8 different CT zones. ${ }^{15}$ Based on these observations, we were able to complete the IOCT-guided location in an acceptable procedural time (mean, 17.1 minutes), which was in line with that obtained in previous series in which IOCT-guided localization was used (mean, 36-39 minutes). ${ }^{12,13,22}$ Notably, IOCT-guided localization in our study was performed by a single team of thoracic surgeons, without involving an interventional radiologist. In addition, and differently from previous studies, all the IOCT-guided localizations were completed in the lateral decubitus position. This approach allowed surgery to be completed as early as possible after completion of localization (ie, without the need for repositioning), with further simplification of the hybrid OR workflow and reduced time at risk.

Besides the procedural success and complication rates, we believe that radiation exposure is an important safety issue that needs to be addressed when different imaging modalities are compared. However, the radiation dynamics of CBCT and MDCT differ significantly, making the direct use of scanner-estimated doses unfeasible for comparing the extent of radiation exposure. A previous study has shown that CBCT used for imaging the abdomen delivers a higher radiation dose compared with MDCT. ${ }^{23}$ However, few data are available for chest imaging, and no study to date has specifically focused on IOCT-guided VATS. In our prospective study, we were able to compare for the first time the individual surface radiation exposure by placing TLDs on the patients. We found no significant intergroup differences in terms of median effective radiation dose between localization by MDCT (POCT group) and CBCT (IOCT group). However, additional 


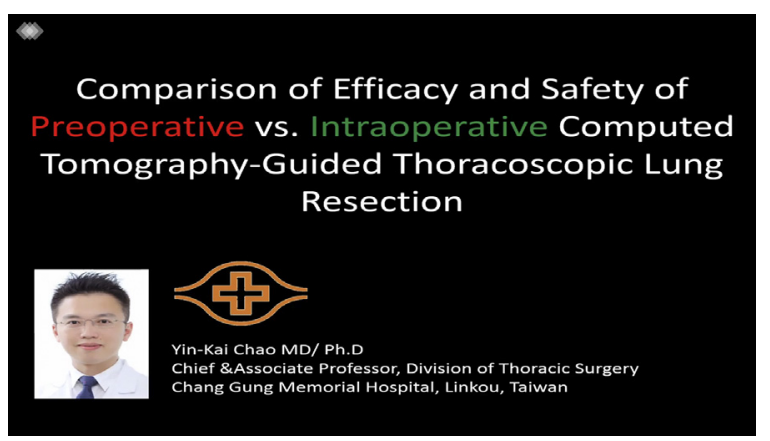

VIDEO 1. Video-assisted thoracoscopic surgery (VATS) is increasingly being used for lung tumor resection owing to more favorable perioperative outcomes and similar survival compared with open surgery. However, its use for treating small or deep or GGN lesions remains challenging. Such lung nodules are frequently thoracoscopically undetectable and not palpable through VATS. In this scenario, the availability of accurate and reliable localization techniques is paramount for allowing lung lesion removal using VATS. For percutaneous CT-guided lung localization, the most common workflow comprises an initial preoperative CT (POCT)-guided lesion localization performed in an interventional CT suite followed by patient transfer to an operating room (OR). Recently, with the increasing availability of hybrid OR, intraoperative CT (IOCT)-guided localization has been proposed to overcome the known shortcomings of the POCT approach. Although there are several studies showing the feasibility of the IOCT approach, a direct comparison between the 2 methods has never been attempted. We therefore designed the current single-center study to address these knowledge gaps. We compared IOCT-guided (IOCT group) and POCT-guided (POCT group) thoracoscopic resection in 64 consecutive patients with undiagnosed lung nodules who required tumor localization between April and October 2017. The main outcome measures included the rate of successful targeting during localization, the time elapsed for (1) tumor localization; (2) surgery preparation time, which was defined as the time between completion of anesthesia and skin incision in POCT group and as the time between completion of anesthesia and $\mathrm{C}$ arm docking plus $\mathrm{C}$ arm parking to skin incision in IOCT group; and (3) time at risk, which was defined as the time between completion of localization to skin incision. We also compared length of time under anesthesia and global operation room utilizing time.

For radiation exposure, 4 sets of thermoluminescent dosimeters were placed around the patient's chest wall at the lesion level. The mean values measured by the dosimeters were used for analysis. All the lung nodules were identifiable on either CBCT or multidetector CT. Localization was successful in all patients. Upon thoracoscopic exploration, failure of localization occurred in 5 patients. There was no significant intergroup differences in successful targeting rate during operation, and no patients required conversion to open thoracotomy. The localization procedural time and radiation exposure were similar between the 2 groups. The use of IOCT significantly reduced the patient time at risk from 215.8 to 13.6 minutes. However, the IOCT group required a longer preparation time in the OR than the POCT group did. The time under general anesthesia and global OR utilization time was also significantly longer in the IOCT group $(P<.001)$. We concluded that comparing with POCT-guided approach, IOCT-guided approach offered similar efficacy and accuracy for lung tumor localization with no increasing in radiation exposure and significantly reduced the time at risk elapsed from localization completion to skin incision. However, the global operation room utilization time was significantly increased. Video available at: https://www. jtcvs.org/article/S0022-5223(18)31856-7/fulltext. phantom studies are needed to compare the degree of organ radiation exposure associated with different interventional approaches.

Despite a lower time at risk when the IOCT-guided approach was used, hybrid OR localization significantly increased both time under anesthesia and global OR utilization time. Notably, the increasing anesthesia time in the IOCT group was not ascribed to the localization procedure per se. Accordingly, we also found that the IOCT group required a significantly longer preparation time before surgery and a longer (albeit not significantly so) operation time than the POCT group did (Figure 4). When the IOCT-guided approach is used, the operating table must be cleared of any protruding add-ons. In addition, the anesthesiology team needs to use sufficiently long cables and tubes to enable a collision-free $\mathrm{C}$-arm rotation for the $\mathrm{CBCT}$ scan. All these procedures increase the complexity of preparation. Notably, the current generation of hybrid OR tables is not specifically designed for thoracic surgery and does not have a hinge joint for bridging. Without flexible tables, the intercostal place cannot be opened up widely (making VATS more difficult and increasing the risk of traumas to the intercostal nerve).

Several caveats of our investigation merit comment. First, patient allocation to IOCT or POCT was not randomized. Because of the limited availability of the hybrid OR for our thoracic surgery service, patients who were scheduled to undergo tumor localization before surgery when the hybrid OR was not available were switched to the POCT-guided technique. Furthermore, the decision to perform tumor localization before VATS was personally taken by the surgeon and not by a multidisciplinary team. We are aware that this could have introduced some selection bias. Second, we did not measure the amount of radiation exposure of the personnel in charge of C-arm CBCT (which is believed to produce a higher amount of scattered radiation compared with MDCT). Third, the IOCT-guided approach significantly increased both the time under anesthesia and the global OR utilization time (theoretically increasing the overall medical costs). Unfortunately, we were unable to provide a more in-depth economic analysis because of the lack of cost data. Fourth, this study was solely focused on the percutaneous CT-guided approach. As a result, we did not compare this method with other non-percutaneous approaches (eg, electromagnetic navigation bronchoscopy or virtual bronchoscopy-guided marker injection). Future head-to-head randomized trials aimed at comparing the efficacy and cost-effectiveness of the available methods are needed to address these issues. ${ }^{24}$ Such studies would provide invaluable data for selecting the most suitable imaging modality for preoperative localization of SPNs before minimally invasive resection. 


\section{CONCLUSIONS}

Compared with the preoperative computed tomographyguided approach, intraoperative computed tomographyguided approach was associated with a decreased time at risk between the completion of localization and skin incision (Video 1). However, the global operating room utilization time increased significantly. Because no significant outcome differences were observed, the choice between the 2 approaches should be based on the most readily available approach at a surgeon's specific facility.

\section{Conflict of Interest Statement}

Authors have nothing to disclose with regard to commercial support.

\section{References}

1. National Lung Screening Trial Research Team, Aberle DR, Adams AM, Berg CD, Black WC, Clapp JD, et al. Reduced lung-cancer mortality with low-dose computed tomographic screening. N Engl J Med. 2011;365:395-409.

2. Suzuki K, Nagai K, Yoshida J, Ohmatsu H, Takahashi K, Nishimura M, et al. Video-assisted thoracoscopic surgery for small indeterminate pulmonary nodules. Chest. 1999;115:563-8.

3. Lin M-W, Tseng Y-H, Lee Y-F, Hsieh M-S, Ko W-C, Chen J-Y, et al. Computed tomography-guided patent blue vital dye localization of pulmonary nodules in uniportal thoracoscopy. J Thorac Cardiovasc Surg. 2016;152:535-44.e532.

4. Ichinose J, Kohno T, Fujimori S, Harano T, Suzuki S. Efficacy and complications of computed tomography-guided hook wire localization. Ann Thorac Surg. 2013; 96:1203-8.

5. Thistlethwaite PA, Gower JR, Hernandez M, Zhang Y, Picel AC, Roberts AC. Needle localization of small pulmonary nodules: Lessons learned. J Thorac Cardiovasc Surg. 2018;155:2140-7.

6. Sato M, Omasa M, Chen F, Sato T, Sonobe M, Bando T, et al. Use of virtual assisted lung mapping (VAL-MAP), a bronchoscopic multispot dye-marking technique using virtual images, for precise navigation of thoracoscopic sublobar lung resection. J Thorac Cardiovasc Surg. 2014;147:1813-9.

7. Sato M, Kuwata T, Yamanashi K, Kitamura A, Misawa K, Imashimizu K, et al. Safety and reproducibility of virtual-assisted lung mapping: a multicentre study in Japan. Eur J Cardiothorac Surg. 2017;51:861-8.

8. Abbas A, Kadakia S, Ambur V, Muro K, Kaiser L. Intraoperative electromagnetic navigational bronchoscopic localization of small, deep, or subsolid pulmonary nodules. J Thorac Cardiovasc Surg. 2017;153:1581-90.

9. Anayama T, Qiu J, Chan H, Nakajima T, Weersink R, Daly M, et al. Localization of pulmonary nodules using navigation bronchoscope and a near-infrared fluorescence thoracoscope. Ann Thorac Surg. 2015;99:224-30.
10. Marino KA, Sullivan JL, Weksler B. Electromagnetic navigation bronchoscopy for identifying lung nodules for thoracoscopic resection. Ann Thorac Surg. 2016;102:454-7

11. Finley RJ, Mayo JR, Grant K, Clifton JC, English J, Leo J, et al. Preoperative computed tomography-guided microcoil localization of small peripheral pulmonary nodules: a prospective randomized controlled trial. J Thorac Cardiovasc Surg. 2015;149:26-32.

12. Kostrzewa M, Kara K, Rathmann N, Tsagogiorgas C, Henzler T, Schoenberg SO et al. Computed tomography-assisted thoracoscopic surgery: a novel, innovative approach in patients with deep intrapulmonary lesions of unknown malignant status. Invest Radiol. 2017;52:374-80.

13. Yang S-M, Ko W-C, Lin M-W, Hsu H-H, Chan C-Y, Wu I-H, et al. Image-guided thoracoscopic surgery with dye localization in a hybrid operating room. J Thorac Dis. 2016;8:S681.

14. Hsieh M-J, Fang H-Y, Lin C-C, Wen C-T, Chen H-W, Chao Y-K. Single-stage localization and removal of small lung nodules through image-guided video-assisted thoracoscopic surgery. Eur J Cardiothorac Surg. 2017 [Epub ahead of print].

15. Hsieh M-J, Wen C-T, Fang H-Y, Wen Y-W, Lin C-C, Chao Y-K. Learning curve of image-guided video-assisted thoracoscopic surgery for small pulmonary nodules: a prospective analysis of thirty initial patients. J Thorac Cardiovasc Surg. 2018;155:1825-32.e1.

16. Chen Y-R, Yeow K-M, Lee J-Y, Su IH, Chu S-Y, Lee C-H, et al. CT-guided hook wire localization of subpleural lung lesions for video-assisted thoracoscopic surgery (VATS). J Formos Med Assoc. 2007;106:911-8.

17. Hsieh C-P, Hsieh M-J, Fang H-Y, Chao Y-K. Imaging-guided thoracoscopic resection of a ground-glass opacity lesion in a hybrid operating room equipped with a robotic C-arm CT system. J Thorac Dis. 2017;9:E416.

18. MacDuff A, Arnold A, Harvey J. Management of spontaneous pneumothorax: British Thoracic Society pleural disease guideline 2010. Thorax. 2010;65:ii18-31.

19. Ujiie H, Kato T, Hu H-p, Patel P, Wada H, Fujino K, et al. A novel minimally invasive near-infrared thoracoscopic localization technique of small pulmonary nodules: a phase I feasibility trial. J Thorac Cardiovasc Surg. 2017;154:702-11.

20. Ohtaka K, Takahashi Y, Kaga K, Senmaru N, Kotani Y, Matsui Y. Video-assisted thoracoscopic surgery using mobile computed tomography: new method for locating of small lung nodules. J Cardiothorac Surg. 2014;9:110.

21. Yendamuri S, Demmy TL. Importance of mapping the external environment in image-guided video-assisted thoracoscopic surgery. J Thorac Cardiovasc Surg. 2018; $155: 1833$.

22. Gill RR, Zheng Y, Barlow JS, Jayender J, Girard EE, Hartigan PM, et al Image-guided video assisted thoracoscopic surgery (iVATS)-phase I-II clinical trial. J Surg Oncol. 2015;112:18-25.

23. Kwok YM, Irani FG, Tay KH, Yang CC, Padre CG, Tan BS. Effective dose estimates for cone beam computed tomography in interventional radiology. Eur Radiol. 2013;23:3197-204.

24. Weksler B. Time for randomization. J Thorac Cardiovasc Surg. 2017;154:712-3.

Key Words: ARTIS zeego, hybrid operating room, localization, solitary pulmonary nodules 
TABLE E1. Size, depth, and location of lung tumors in the 64 study patients

\begin{tabular}{|c|c|c|c|c|c|}
\hline \multirow[b]{2}{*}{ Depth size } & \multicolumn{2}{|c|}{ Ground-glass nodules } & \multicolumn{2}{|c|}{ Solid nodules } & \multirow[b]{2}{*}{ Total } \\
\hline & $\leq \mathbf{1 0} \mathbf{~ m m}$ & $>10 \mathrm{~mm}$ & $\leq \mathbf{1 0} \mathbf{~ m m}$ & $>10 \mathrm{~mm}$ & \\
\hline$\leq 10 \mathrm{~mm}$ & $15(\mathbf{1 1} / 4)$ & $13(4 / 9)$ & $21(\mathbf{1 5} / 6)$ & $7(2 / 5)$ & $56(32 / 24)$ \\
\hline$>10 \mathrm{~mm}$ & $0(0 / 0)$ & $4(0 / 4)$ & $2(0 / 2)$ & $2(0 / 2)$ & $8(0 / 8)$ \\
\hline Total & $15(\mathbf{1 1} / 4)$ & $17(4 / 13)$ & $23(\mathbf{1 5} / 8)$ & $9(\mathbf{2} / 7)$ & $64(32 / 32)$ \\
\hline
\end{tabular}

The numbers within brackets indicate the localization method (dye/wire). 